\title{
Implementation of an Innovative Method to Design Reflectarray Antennas
}

\author{
Abdelhamid Tayebi, Josefa Gómez, Iván González, and Felipe Cátedra \\ Electromagnetic Computing Group, Computer Science Department, University of Alcalá, Escuela Politécnica, \\ Alcalá de Henares, 28871 Madrid, Spain \\ Correspondence should be addressed to Abdelhamid Tayebi, hamid.tayebi@uah.es
}

Received 2 March 2012; Revised 16 May 2012; Accepted 5 June 2012

Academic Editor: Sandra Costanzo

Copyright ( $\odot 2012$ Abdelhamid Tayebi et al. This is an open access article distributed under the Creative Commons Attribution License, which permits unrestricted use, distribution, and reproduction in any medium, provided the original work is properly cited.

\begin{abstract}
A novel computed aided technique for designing reflectarray antennas is presented. The developed approach automatically generates the geometrical model of reflectarray antennas taking into account some input parameters, such as, the unit cell type and dimensions, frequency, focal length, periodicity, dielectric materials, and desired main beam radiating direction. The characteristics of the reflecting elements are selected considering the spatial phase delay at each unit cell to achieve a progressive phase shift. The implemented procedure also provides the phase characteristic of the unit element, which is rapidly computed by using a parallelized Moment Method (MoM) approach. The MoM is also used to obtain the radiation pattern of the full reflectarray antenna. In order to evaluate the new technique, a dual-interface prototype has been designed and simulated showing high-performance capability.
\end{abstract}

\section{Introduction}

Reflectarray antennas [1] have become an attractive alternative to the traditional parabolic reflector antennas due to some advantages, such as, light weight, mechanical robustness, compatibility with active devices, simple manufacturing, low profile, and low cost. Moreover, reflectarray antennas offer the possibility of beam steering, like conventional phased arrays, but eliminate the complexity and losses of the feeding network, showing a higher efficiency. Their main limitation is the narrow-band behavior, which is inherent to the printed antenna structures. To overcome this shortcoming, some proposals based on multilayer configurations $[2,3]$ and concentric rings $[4,5]$ have been reported lately. For instance, the design reported in [2] is composed of microstrip stacked patches and shows good performance in dual frequency operation. The reflectarray presented in [3] consists of three stacked layers with rectangular patches and provides dual-polarization operation for space applications. Although the bandwidth is notably increased when using multilayer printed patches, other issues may arise, such as, the manufacturing complexity and higher weight and losses. On the other hand, reflectarrays based on concentric rings are also becoming very popular due to they provide excellent results using one-layer configurations. The schemes proposed in [4] achieve a significant improvement in gainbandwidth using double square and double cross loop elements. A comparable elementary cell that contains double square and double circular rings is studied in [5], where the phasing characteristic of the single-layer cell is computed showing good results.

Reflectarray antennas are composed of one or more layers of metallic elements and dielectric substrates over a ground plane. They are typically fed by a horn antenna and their operation consists of converting a spherical wavefront into a desired scattered wavefront using a phase shift mechanism. Although it has been demonstrated that reflectarray antennas can be used to generate contoured beams [6], most of them are still designed to radiate a collimate wave front at a certain frequency. To achieve this, according to Figure 1, each 


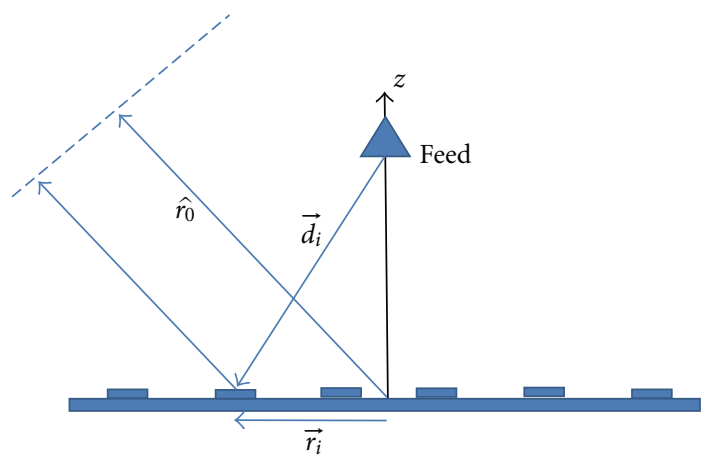

Figure 1: Diagram of the reflectarray antenna.

periodic cell must introduce a phase shift that is obtained as follows:

$$
\phi_{i}=k_{0}\left(d_{i}-\overrightarrow{r_{i}} \cdot \hat{r_{0}}\right)+2 \pi N ; \quad N=0,1,2, \ldots,
$$

where $k_{0}$ is the propagation constant in vacuum, $\widehat{r_{0}}$ is the unit vector in the desired direction of the main beam, $\vec{r}_{i}$ is the position vector from the center of the reflectarray plane to the $i$ th radiating element, and $d_{i}$ is the distance from the feed to the ith element.

Hence, the resonant size, periodicity, orientation, and substrate's properties of every cell must be properly selected to provide the phase shift given by (1).

Due to the radiating elements must reflect the incident electromagnetic field with a progressive phase distribution, the most challenging aspect when designing reflectarray antennas is to find the adequate phase shift to compensate the phase delay caused by the distance from the feed to each unit cell. Thus, the requirement of having a wide database that associates the properties of the cells and their correspondent phase shifts is crucial. In fact, many works proposing different techniques to achieve a wide phase range of the reflection coefficient have been recently published. In the literature, mainly four mechanisms can be found. The first one is the easiest to manufacture and more used $[7,8]$. It achieves the phase variation by changing the size of the internal geometry of the unit cells. The second type tries to find the proper phase shift by adjusting the rotation angle of the elements [9]. The third method to provide the progressive phase shift of the reflected wave consists of using variable stub length attached to the radiating elements [10]. Finally, the fourth mechanism consists in using multilayer configurations $[2,3]$. The proposed method applies these four techniques, since it is able to find the suitable characteristics of each elementary cell.

Other important aspect is the adequate choice of the radiating element's shape, since the scatter of the incident field strongly depends on its geometry. Hence, an essential goal is to obtain the type of cell that provides a wide reflection coefficient phase range when varying its properties. In fact, it is well-known that a reduced phase range lower than $360^{\circ}$ implies a decreasing in directivity. On the other hand, it has been demonstrated $[11,12]$ that the phase variation depending on any geometrical parameter should be as lineal as possible, providing a smooth slope. This implies that good results are provided only if the $360^{\circ}$ range is achieved by varying the considered parameter extremely slowly. In practical situations, the consequences of abrupt variations in the phase are narrower bandwidths and a more complicated manufacturing of the radiation elements due to the minimum tolerances.

Normally, the reflection coefficient phase as a function of the patch size is calculated to determine whether the phase range is wide enough and the curve slope is smooth enough. Thus, a lot of effort is usually required to find a unit cell able to provide the mentioned phase characteristic. The traditional shapes of microstrip patches have been crosses, squares, rectangles, rings, and circles. In this paper, the double square ring has been chosen to prove the efficiency of the developed computer tool.

A new method to automatically design reflectarray antennas is proposed. The properties of the radiating elements, including their resonant dimensions and substrate's characteristics, are computed by means of an efficient process that compensates the phase shift introduced by each elementary cell. Although several works have been recently carried out to investigate the phase characteristics of the cell elements, most of them do not report the reflectarray design once the phase curve has been properly achieved. The contribution of this paper also relies on the final design of the reflectarray, besides implementing the new approach and obtaining the widely used phase curve of the elementary cell. The proposed approach has been included in the computer tool NEWFASANT [13] to validate its performance.

The paper is organized as follows. The procedure to obtain the geometrical model of the reflectarray is described in Section 2, the design of a reduced-scale prototype is stated in Section 3, and finally some conclusions are remarked in Section 4.

\section{Design Process}

The basic idea of the new technique is the automatic design of reflectarray antennas by varying the parameters of the unit cells. The variation may include the resonant size of the cells, their position, orientation, stub's length, periodicity, number of layers, dielectric materials, and so forth, so that the phase shift given by every element is compensated according to the phase of the reflection coefficient.

First, a database which stores the values of the modified parameters and its correspondent reflection coefficient phase is created at a given frequency and for a determined periodicity. It is important to highlight that the analysis can be conducted by varying one or more parameters. Each entry in the database that associates pairs of parameter values and reflection phases is obtained by analyzing the behavior of a quasi-infinite array of identical elements by applying a full wave method based on the MoM [14], so truncation effects due to the finite size of real reflectarrays are also considered. Despite the big computational effort required to obtain the whole database, it is affordable in time because the MoM code is parallelized. A normal incident plane wave 


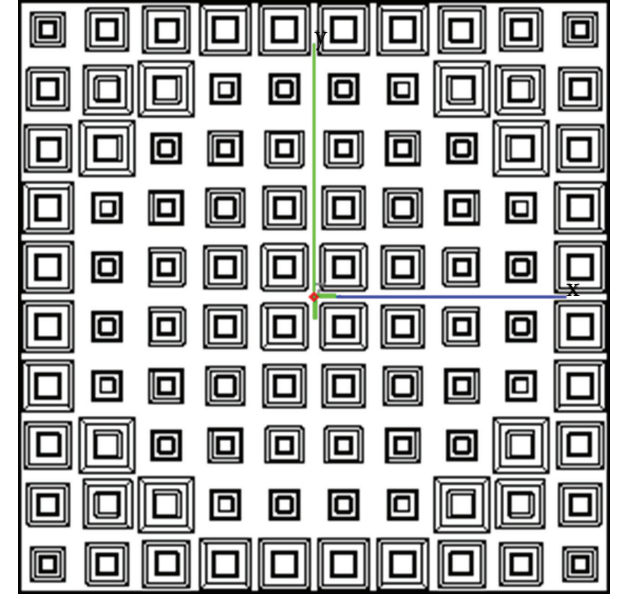

Figure 2: Top view of the reflectarray antenna.

is considered to compute the reflection coefficient phases. However, due to the fact that the radiation of the real feed antenna has an incidence direction with a certain angle, a further refinement can be done considering some incidence angles of the planar wave. In that case, the tool is able to provide as many databases as incidence angles are indicated.

Then, the phase delay caused by the path difference from the feed point is calculated taking into account the position of each unit cell. Once all phase delays are obtained, the system searches in the database the proper dimensions to compensate the required phase at every element. Hence, each cell can properly adjust their phase delay, introducing no errors and obtaining the maximum directivity. The phase shift needed in each cell when using several incident angles is obtained from one of those databases depending on its position and the feed location. The results provided considering several incident angles are slightly more accurate than only considering normal incidence. The next step consists in creating the geometrical model of the reflectarray, according to the values provided by the database. Finally, the ground plane is correctly located under the cells conforming to the dielectric thicknesses. By default, the reflectarray is created in the $X Y$ plane, but it is possible to fit it to curved surfaces. The user is also able to define the shape of the reflectarray, which can be rectangular, square, circular, or elliptical. When the reflectarray has been designed and we have the antenna geometry, the MoM is applied again to obtain a rigorous solution considering the full reflectarray antenna.

The flexibility when designing reflectarray antennas by using the presented tool is enormous, since many combinations of different shapes, dimensions, diverse substrates, incidences, and so forth can be used to design the radiating elements. In addition, the configuration of the unit cell is not constrained to a single layer. In fact, the tool provides a great versatility and allows defining various layers of metallic patches and interfaces of different substrates. Moreover, in order to facilitate the use of the implemented tool, several databases that contain correspondences between dimensions and reflection coefficient phases of many elementary cells

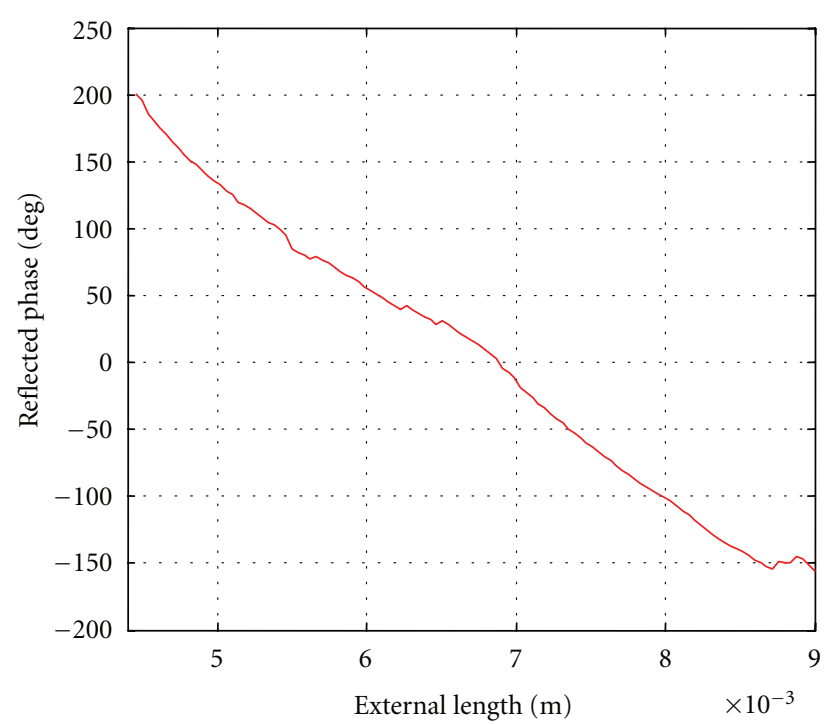

Figure 3: Phase characteristic of the unit cell varying the length of the external square ring.

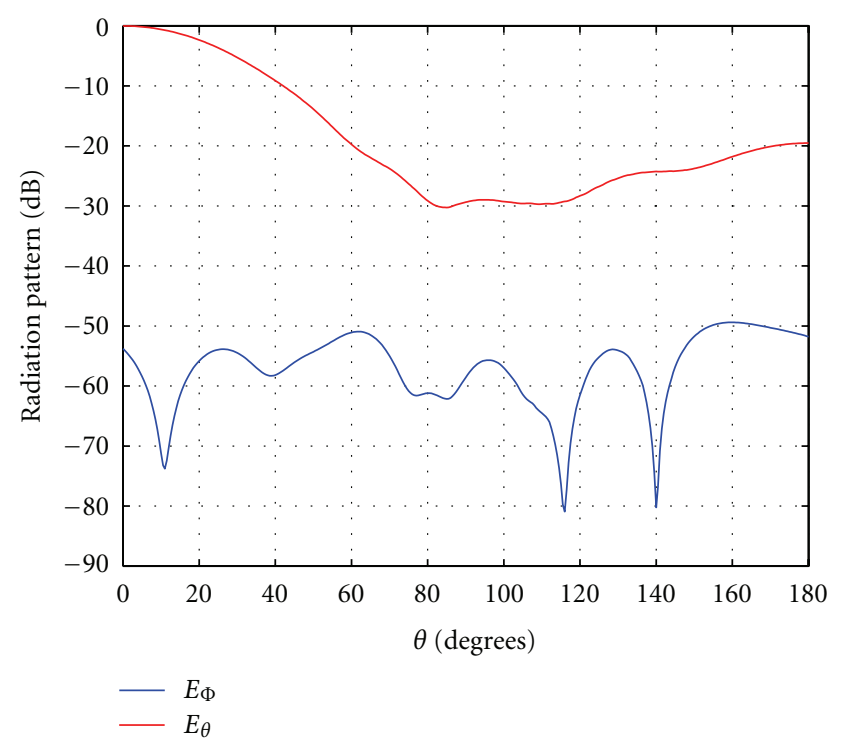

Figure 4: Radiation pattern of the feed antenna at $20 \mathrm{GHz}$.

(rectangular, circular and square patches, square and circular rings, metallic crosses, patches with holes, etc.) are available.

The module includes three techniques to model the feed antenna. The first one allows importing the radiation pattern of a real horn antenna. The second one allows importing the geometrical model of the feed antenna in several CAD formats, and the third one allows creating the physical model of the feed antenna.

\section{Validation of the Implemented Tool}

In order to validate the performance of the presented method, the specifications of an existing design recently published [15] have been considered. The reflectarray has 


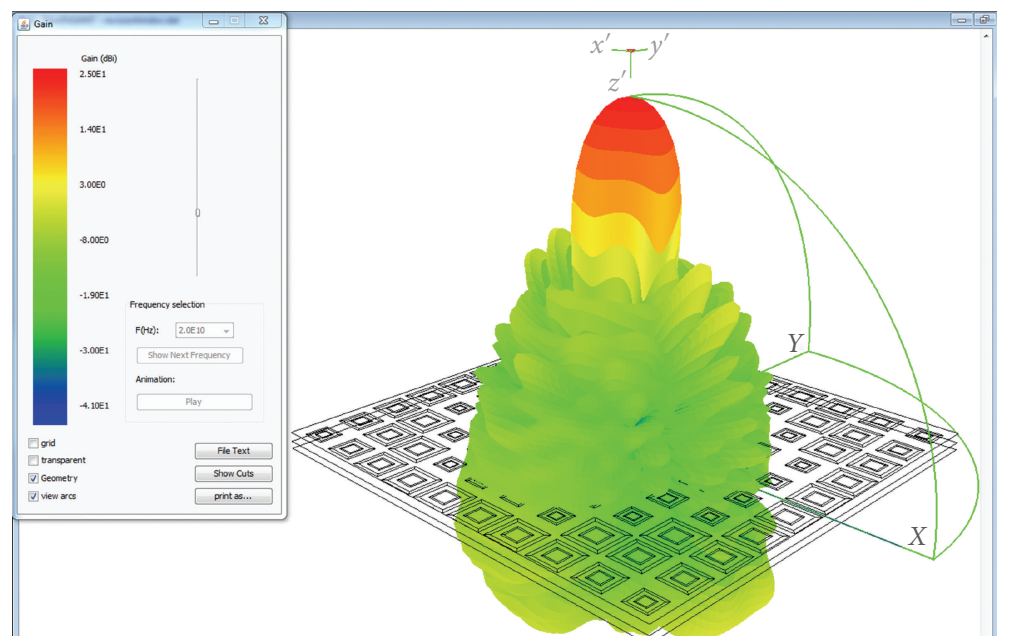

Figure 5: Radiation pattern in 3D at $20 \mathrm{GHz}$.

been redesigned using the propose procedure that adjusts the dimensions of the patches to focus or the main beam in the normal direction at $20 \mathrm{GHz}$. In our case, it is assumed that the antenna is composed of 100 identical unit cells symmetrically located in a square grid with periodicity of $9 \mathrm{~mm}$. The top view is depicted in Figure 2.

Figure 3 shows the phase variation versus the length of the external square ring that has been automatically provided by our tool, [13]. The complete range of $360^{\circ}$ is achieved by varying the length of the external square ring from $4.5 \mathrm{~mm}$ to $9 \mathrm{~mm}$. It can be observed that the slope is practically lineal. These results have been achieved using a $2.95 \mathrm{~mm}$ substrate of foam under the metallic rings and a $0.85 \mathrm{~mm}$ layer of a material whose relative dielectric constant is 2.5 (same multilayer structure than in [15]). This thin layer is located on the cells, acting as a protective surface. On the other hand, the width of both rings and the gap between them have been fixed to $0.3 \mathrm{~mm}$ independently of the length variation.

As in [15], the reflectarray antenna is fed by means of a linearly polarized pyramidal horn located at $x=0, y=0$, $z=0.0712 \mathrm{~m}$. The normalized radiation pattern of the fed antenna is depicted in Figure 4 at the central frequency of $20 \mathrm{GHz}$.

On the other hand, Figure 5 shows the 3D radiation pattern of the reflectarray antenna we have redesigned, where the main beam radiating in the normal direction is clearly depicted. Regarding the polarization purity, Figure 6 shows the cut $\varphi=0^{\circ}$ of the normalized radiation pattern at $20 \mathrm{GHz}$, where it can be observed that the cross-polar component is lower than $-30 \mathrm{~dB}$ for every $\theta$ value. The second side lobes are also quite low, under $-20 \mathrm{~dB}$.

Finally, Figure 7 depicts the gain provided by the redesigned reflectarray. Note that the maximum value is $23.5 \mathrm{dBi}$, which is higher than the value of $20 \mathrm{dBi}$ achieved in [15]. It is important to point out that the analysis of the final reflectarray has been conducted by using the MoM module of NEWFASANT, although it could have been used a different electromagnetic solver. Since reflectarray antennas are electrically very large structures, the multilevel fast

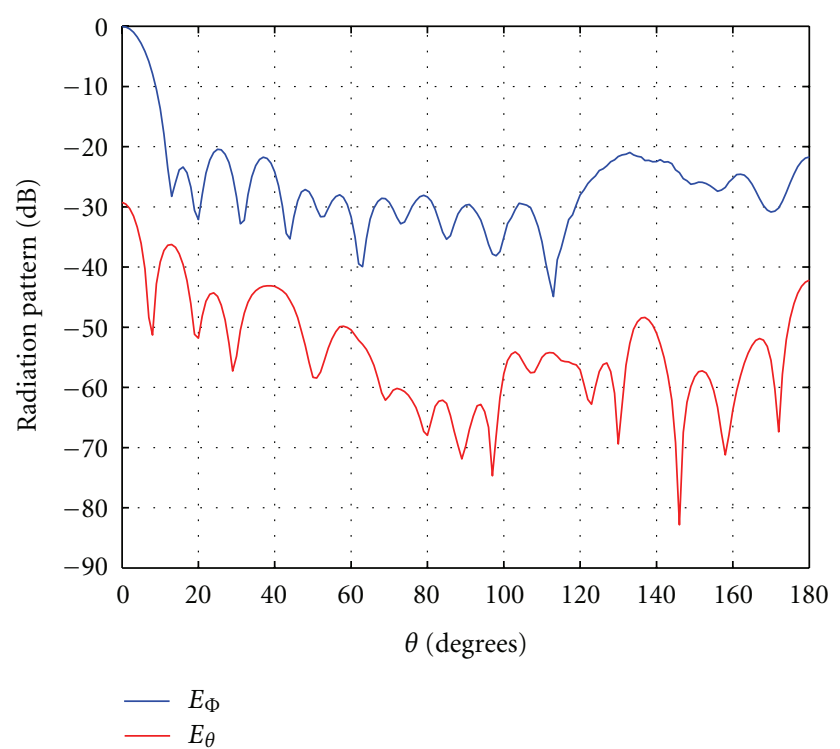

FIGURE 6: Normalized radiation pattern. Cut $\varphi=0^{\circ}$, frequency $=$ $20 \mathrm{GHz}$.

multipole algorithm is used in the full wave analysis to reduce the required memory and time resources.

\section{Conclusion}

A powerful tool to automatically design reflectarray antennas has been presented. The reported technique is able to generate the geometrical model of the antenna considering some input parameters, such as, the unit cell type, the operating frequency, the focal length, the periodicity, and the desired main beam radiating direction. The tool also computes and provides the phase curve of the unit cell. To evaluate the performance of the new method, a recently published reflectarray has been redesigned. Excellent results 


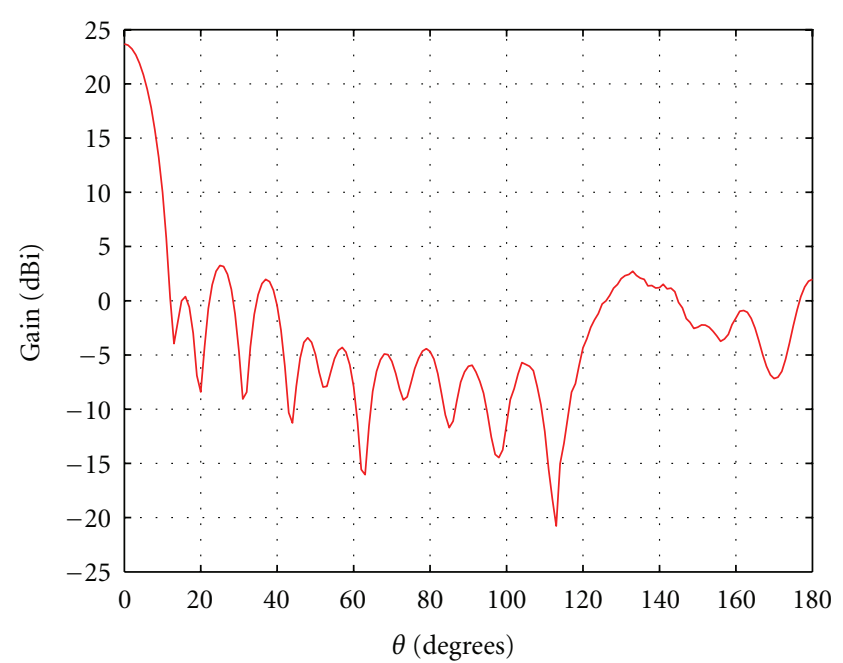

FIgURe 7: Gain. Cut $\varphi=0^{\circ}$, frequency $=20 \mathrm{GHz}$.

in terms of gain and polarization purity have been achieved. The developed tool can be very helpful for researchers.

\section{Acknowledgments}

This work has been supported, in part by the Comunidad de Madrid Project S-2009/TIC1485 and by the Castilla-La Mancha Project PPII10-0192-0083, by the Spanish Department of Science, Technology Projects TEC 2010-15706 and CONSOLIDER-INGENIO No CSD-2008-0068.

\section{References}

[1] D. Berry, R. Malech, and W. Kennedy, "The reflectarray antenna," IEEE Transactions on Antennas and Propagation, vol. 11, no. 6, pp. 645-651, 1963.

[2] J. A. Encinar, "Design of a dual frequency reflectarray using microstrip stacked patches of variable size," Electronics Letters, vol. 32, no. 12, pp. 1049-1050, 1996.

[3] G. Zhao and Y.-C. Jiao, "Broadband dual-polarization dualcoverage reflectarray antenna," in Proceedings of the IEEE 4th International Symposium on Microwave, Antenna, Propagation, and EMC Technologies for Wireless Communications (MAPE '11), pp. 102-105, November 2011.

[4] M. R. Chaharmir, J. Shaker, M. Cuhaci, and A. Ittipiboon, "A broadband reflectarray antenna with double square rings as the cell elements," in Proceedings of the European Conference on Antennas and Propagation (EuCAP '06), pp. 1-4, November 2006.

[5] M. E. Bialkowski and K. H. Sayidmarie, "Phasing characteristics of variable size double rings of square or circular shape for design of a single layer microstrip reflectarray," in Proceedings of the 17th International Conference on Microwaves, Radar and Wireless Communications (MIKON '08), pp. 1-4, May 2008.

[6] J. A. Encinar, L. S. Datashvili, J. A. Zornoza et al., "Dual-polarization dual-coverage reflectarray for space applications," IEEE Transactions on Antennas and Propagation, vol. 54, no. 10, pp. 2828-2837, 2006.

[7] J. A. Encinar, "Analysis and design of dual frequency reflectarrays using microstrip stacked patches of variable size," in
Proceedings of the 26th European Microwave Conference, vol. 1, pp. 221-224, September 1996.

[8] J. A. Encinar, "Design of two-layer printed reflectarrays using patches of variable size," IEEE Transactions on Antennas and Propagation, vol. 49, no. 10, pp. 1403-1410, 2001.

[9] M. E. Cooley, J. F. Walker, D. G. Gonzalez, Pollon, and G. E, "Novel reflectarray element with variable phase characteristics," IEE Proceedings, vol. 144, no. 2, pp. 149-151, 1997.

[10] R. D. Javor, X. D. Wu, and K. Chang, "Design and performance of a microstrip reflectarray antenna," IEEE Transactions on Antennas and Propagation, vol. 43, no. 9, pp. 932-939, 1995.

[11] M. E. Bialkowski and K. H. Sayidmarie, "Investigations into phase characteristics of a single-layer reflectarray employing patch or ring elements of variable size," IEEE Transactions on Antennas and Propagation, vol. 56, no. 11, pp. 3366-3372, 2008.

[12] P. Krachodnok and R. Wongsan, "Phase-bandwidth enhancement of microstrip patch reflectarray with cross slot loads," in Proceedings of the Asia-Pacific Microwave Conference (APMC '07), pp. 1-4, December 2007.

[13] http://www.fasant.com/.

[14] F. Cátedra, C. Delgado, E. García, and I. González, “Combination of MLFMA and an iterative method to compute large scattering or radiation problems," in Proceedings of the 4th European Conference on Antennas and Propagation (EuCAP '10), Barcelona, Spain, April 2010.

[15] B. Ma, L. Yan, M. Xia, C. Wang, and L. Cui, "Design of reflectarray antenna using double square ring elements," in Proceedings of the 2nd IEEE International Conference on Microwave Technology and Computational Electromagnetics (ICMTCE '11), pp. 213-216, May 2011. 

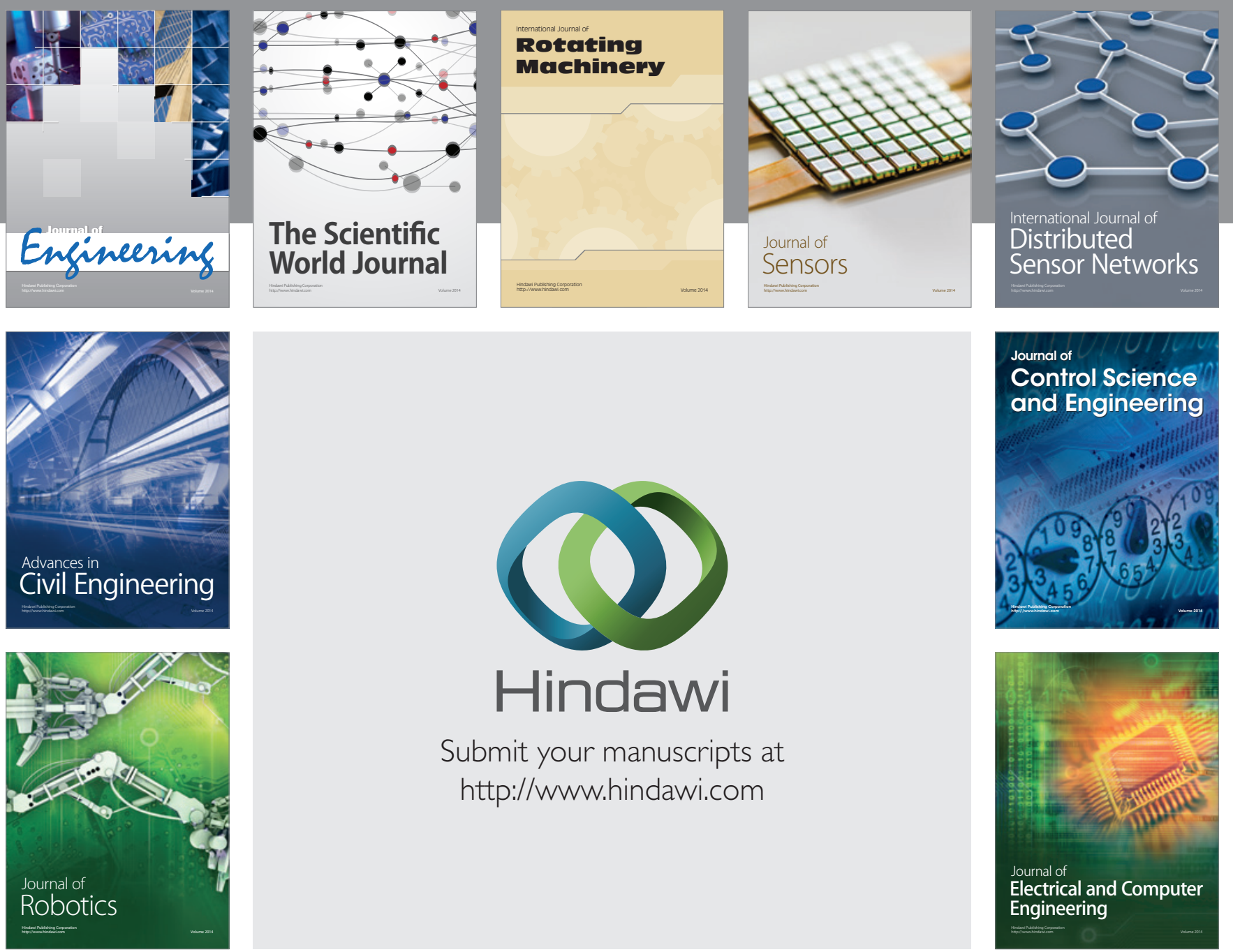

Submit your manuscripts at

http://www.hindawi.com
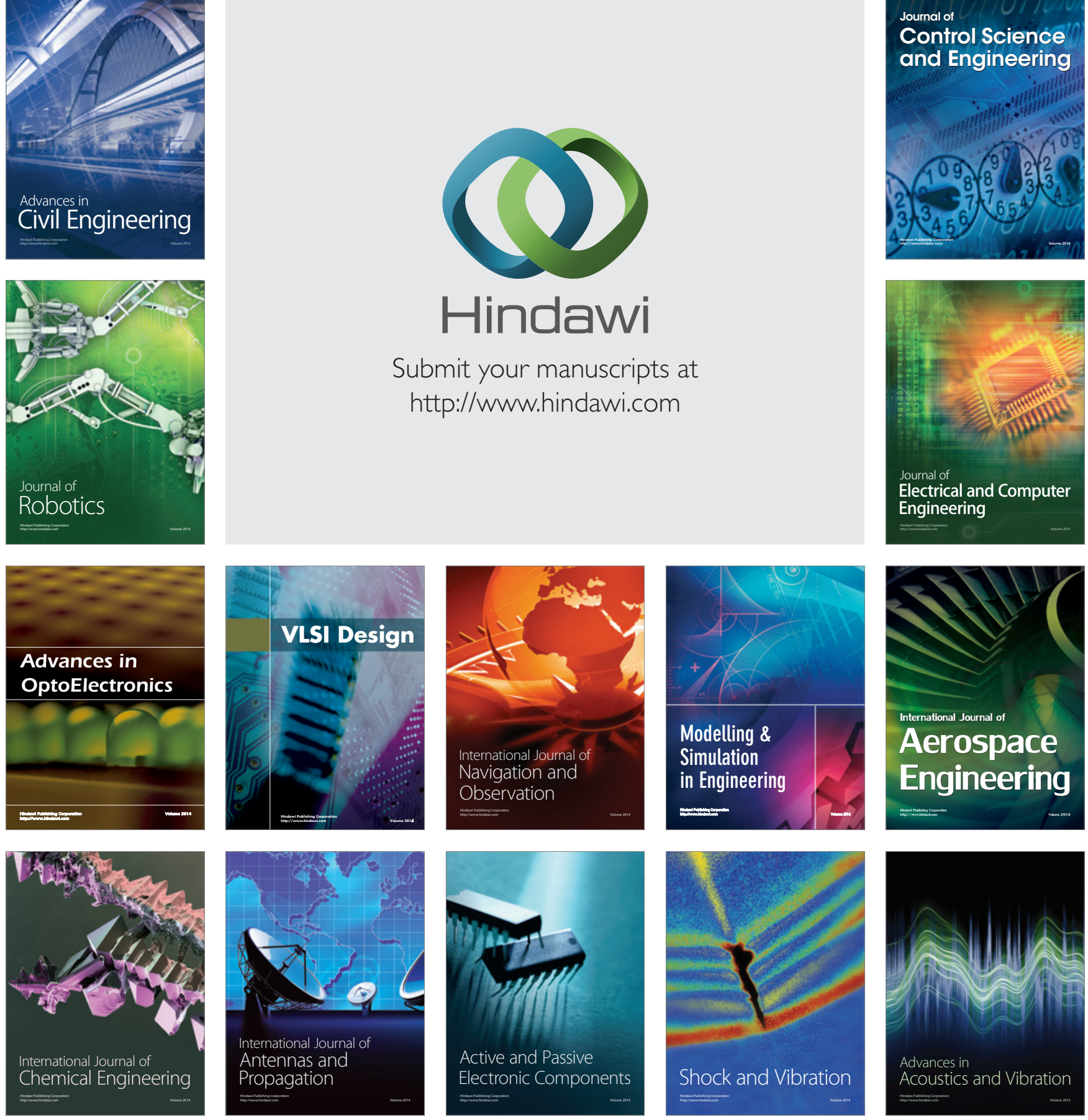